\title{
A Comparative Study of Real-Time RT-PCR-Based SARS-CoV-2 Detection Methods and Its Application to Human-Derived and Surface Swabbed Material
}

Tastanova, Aizhan ; Stoffel, Corinne Isabelle ; Dzung, Andreas ; Cheng, Phil Fang ; Bellini, Elisa ; Johansen, Pål ; Duda, Agathe ; Nobbe, Stephan ; Lienhard, Reto ; Bosshard, Philipp Peter ; Levesque, Mitchell Paul

\begin{abstract}
Real-time RT-PCR remains a gold standard in the detection of various viral diseases. In the coronavirus 2019 pandemic, multiple RT-PCR-based tests were developed to screen for viral infection. As an emergency response to increasing testing demand, we established a severe acute respiratory syndrome coronavirus 2 (SARS-CoV-2) PCR diagnostics platform for which we compared different commercial and in-house RT-PCR protocols. Four commercial, one customized, and one in-house RT-PCR protocols were evaluated with 92 SARS-CoV-2-positive and 92 SARS-CoV-2-negative samples. Furthermore, economical and practical characteristics of these protocols were compared. In addition, a highly sensitive digital droplet PCR (ddPCR) method was developed, and application of RT-PCR and ddPCR methods on SARS-CoV-2 environmental samples was examined. Very low limits of detection (1 or 2 viral copies/ L), high sensitivities (93.6\% to $97.8 \%$ ), and high specificities (98.7\% to 100\%) for the tested RT-PCR protocols were found. Furthermore, the feasibility of downscaling two of the commercial protocols, which could optimize testing capacity, was demonstrated. Tested commercial and customized RT-PCR detection kits show very good and comparable sensitivity and specificity, and the kits could be further optimized for use on SARS-CoV-2 viral samples derived from human and surface swabbed samples.
\end{abstract}

DOI: https://doi.org/10.1016/j.jmoldx.2021.04.009

Posted at the Zurich Open Repository and Archive, University of Zurich

ZORA URL: https://doi.org/10.5167/uzh-203777

Journal Article

Published Version

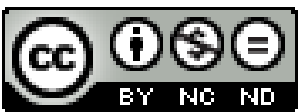

The following work is licensed under a Creative Commons: Attribution-NonCommercial-NoDerivatives 4.0 International (CC BY-NC-ND 4.0) License.

Originally published at:

Tastanova, Aizhan; Stoffel, Corinne Isabelle; Dzung, Andreas; Cheng, Phil Fang; Bellini, Elisa; Johansen, Pål; Duda, Agathe; Nobbe, Stephan; Lienhard, Reto; Bosshard, Philipp Peter; Levesque, Mitchell Paul (2021). A Comparative Study of Real-Time RT-PCR-Based SARS-CoV-2 Detection Methods and Its Application to Human-Derived and Surface Swabbed Material. Journal of Molecular Diagnostics, 23(7):796-804. 
DOI: https://doi.org/10.1016/j.jmoldx.2021.04.009 


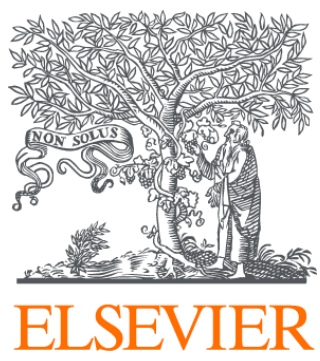

Since January 2020 Elsevier has created a COVID-19 resource centre with free information in English and Mandarin on the novel coronavirus COVID-

19. The COVID-19 resource centre is hosted on Elsevier Connect, the company's public news and information website.

Elsevier hereby grants permission to make all its COVID-19-related research that is available on the COVID-19 resource centre - including this research content - immediately available in PubMed Central and other publicly funded repositories, such as the WHO COVID database with rights for unrestricted research re-use and analyses in any form or by any means with acknowledgement of the original source. These permissions are granted for free by Elsevier for as long as the COVID-19 resource centre remains active. 


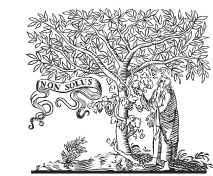

ELSEVIER

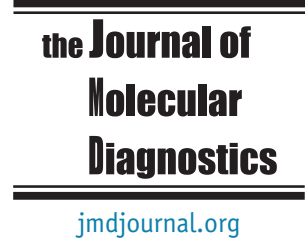

\section{A Comparative Study of Real-Time RT-PCR-Based SARS-CoV-2 Detection Methods and Its Application to Human-Derived and Surface Q1 Swabbed Material}

Q12 Aizhan Tastanova, ${ }^{*}$ Corinne Isabelle Stoffel, ${ }^{*}$ Andreas Dzung, ${ }^{*}$ Phil Fang Cheng, ${ }^{*}$ Elisa Bellini, ${ }^{*}$ Pål Johansen, ${ }^{*}$ Agathe Duda, Stephan Nobbe, ${ }^{*}$ Reto Lienhard, ${ }^{\dagger}$ Philipp Peter Bosshard, ${ }^{*}$ and Mitchell Paul Levesque*

From the Department of Dermatology, * University Hospital Zurich, University of Zurich, Zurich; and ADMed Microbiologie,$^{\dagger}$ La Chaux-de-Fonds, Switzerland

Accepted for publication April 21, 2021.

Address correspondence to Mitchell Paul Levesque, Department of Dermatology, University Hospital Zurich, University of Zurich, Wagistrasse 18, Schlieren, Zürich CH8952, Switzerland. E-mail: mitchell.levesque@usz.ch.

\begin{abstract}
Real-time RT-PCR remains a gold standard in the detection of various viral diseases. In the coronavirus 2019 pandemic, multiple RT-PCR-based tests were developed to screen for viral infection. As an emergency response to increasing testing demand, we established a severe acute respiratory syndrome coronavirus 2 (SARS-CoV-2) PCR diagnostics platform for which we compared different commercial and in-house RT-PCR protocols. Four commercial, one customized, and one in-house RT-PCR protocols were evaluated with 92 SARS-CoV-2-positive and 92 SARS-CoV-2-negative samples. Furthermore, economical and practical characteristics of these protocols were compared. In addition, a highly sensitive digital droplet PCR (ddPCR) method was developed, and application of RT-PCR and ddPCR methods on SARS-CoV-2 environmental samples was examined. Very low limits of detection (1 or 2 viral copies/ $\mu \mathrm{L})$, high sensitivities (93.6\% to $97.8 \%)$, and high specificities $(98.7 \%$ to $100 \%)$ for the tested RT-PCR protocols were found. Furthermore, the feasibility of downscaling two of the commercial protocols, which could optimize testing capacity, was demonstrated. Tested commercial and customized RT-PCR detection kits show very good and comparable sensitivity and specificity, and the kits could be further optimized for use on SARS-CoV-2 viral samples derived from human and surface swabbed samples. (J Mol Diagn 2021, $\mathbf{\square}:$ 1-9; https://doi.org/10.1016/ j.jmoldx.2021.04.009)
\end{abstract}

Q5 On March 11, 2020, the World Health Organization (WHO)

Q6 (Geneva, Switzerland) declared a pandemic because of the quick spread of a respiratory disease caused by the novel severe acute respiratory syndrome coronavirus 2 (SARSCoV-2). With cases increasing in multiple countries and high transmissibility of SARS-CoV-2, eradication is rather unrealistic in the short term. ${ }^{1}$ In Switzerland, the second wave of SARS-CoV-2 is predicted to be slower than the first one but with a higher case fatality rate. ${ }^{2}$ The same situation was reported by the WHO for Spanish influenza for which the second and third waves of the infection claimed more lives and the pandemic lasted for almost 2 years and resulted in at least 50 million deaths worldwide [Centers for Disease
Control and Prevention (CDC), https://www.cdc.gov/flu/ pandemic-resources/1918-commemoration/three-waves.htm, last accessed September 7, 2020]. Another important factor contributing to the rapid spread of the coronavirus disease 2019 (COVID-19) pandemic is an unusually high number

Supported by University Hospital Zurich Innovation Fund grant Q2 INOV00093 (M.P.L.) and Innosuisse grant 46938.1 INNO-LS for the development of the digital droplet PCR assay.

A.T. and C.I.S. contributed equally to this work. P.P.B. and M.P.L. contributed equally to this work as senior authors.

Disclosures: M.P.L. is a founder and shareholder of Oncobit, which partially funded the establishment of the novel real-time RT-PCR and digital droplet PCR assays. 
Applied Biosystems

Multiplex TaqMan 2019nCoV Assay Kit version 2 (research use only) kit of asymptomatic spreaders. ${ }^{3,4}$ Therefore, continuous testing and reliable detection of the virus are essential parts of controlling the spread of SARS-CoV-2 (WHO, https://www. who.int/emergencies/diseases/novel-coronavirus-2019/strate gies-and-plans, last accessed September 7, 2020).

In March 2020, an in-house platform for SARS-CoV-2 diagnostics was initiated as part of an emergency response to an increasing demand for test capacity in a routine microbiology laboratory at University Hospital in Zurich, Switzerland. Currently, the gold standard for the detection and diagnosis of SARS-CoV-2 infection is based on the real-time RT-PCR. The overall goal was to provide in-house SARS-CoV-2 diagnosis to all patients and personnel to ensure the safe and efficient continuation of the health care work within the hospital and the protection of high-risk patients. The aims of this study were i) to evaluate four commercially available, one customized, and one in-house RT-PCR test by comparing the limit of detection (LoD), sensitivity using a panel of SARS-CoV-2 confirmed cases, and specificity using a group of non-COVID-19 respiratory samples; ii) to examine the feasibility of down-scaling two commercial protocols to optimize the testing capacity; iii) to develop a droplet digital PCR (ddPCR) assay to increase test sensitivity and provide more accurate quantitation of viral RNA; and iv) to examine applicability of two validated RT-PCR protocols as well as of a ddPCR protocol on SARSCoV-2 environmental samples.

Table 1 Description of Real-Time RT-PCR Assays Compared in the Study

\begin{tabular}{|c|c|c|c|c|}
\hline RT-PCR protocol & $\begin{array}{l}\text { Abbreviated } \\
\text { name }\end{array}$ & RT-PCR kit/primer and probes & Mastermix used in this study & Positive control \\
\hline $\begin{array}{l}\text { CDC 2019-Novel Coronavirus } \\
\text { Real-Time RT-PCR } \\
\text { Diagnostic Panel (for } \\
\text { in vitro diagnostic }\end{array}$ & $\operatorname{CDC}$ & $\begin{array}{l}\text { 2019-nCoVEUA-01 Diagnostic } \\
\text { Panel Box, catalog number } \\
\text { 10006606, IDT, Newark, NJ }\end{array}$ & $\begin{array}{l}\text { TaMan, Fast Virus 1-step } \\
\text { Maste Mix, 4444436, } 10 \\
\text { mL, Applied Biosystems/ } \\
\text { Thermo Fisher Scientific, } \\
\text { Waltham, MA }\end{array}$ & $\begin{array}{l}\text { 2019- } \\
\text { nCoV_N_Positive } \\
\text { Control, catalog } \\
\text { number } \\
\text { 10006625, IDT }\end{array}$ \\
\hline $\begin{array}{l}\text { EURORealTime SARS-CoV-2 } \\
\text { (for research use only) }\end{array}$ & Euroimmun & Catalog number MP 2606-0425 & Provided with the kit & $\begin{array}{l}\text { Provided with the } \\
\text { kit }\end{array}$ \\
\hline $\begin{array}{l}\text { Real-time RT-PCR assays for } \\
\text { the detection of SARS- } \\
\text { CoV-2, } \\
\text { Pasteur Institute, Paris, } \\
\text { France }\end{array}$ & $\begin{array}{l}\text { Pasteur } \\
\text { Institute } \\
\text { Protocol Pairs } \\
\text { (WHO) }\end{array}$ & $\begin{array}{l}\text { https://www.who.int/docs/ } \\
\text { default-source/coronaviruse/ } \\
\text { real-time-rt-pcr-assays-for-the- } \\
\text { detection-of-sars-cov-2-institut- } \\
\text { pasteur-paris.pdf, last accessed } \\
\text { November 12, 20206 ; ordered } \\
\text { from Microsynth (Balgach, } \\
\text { Switzerland) }\end{array}$ & $\begin{array}{l}\text { Invitrogen Superscript III } \\
\text { Platinum One-Step } \\
\text { quantitative RT-PCR } \\
\text { system, catalog number } \\
\text { 11732-088 }\end{array}$ & $\begin{array}{l}\text { Available on } \\
\text { request from the } \\
\text { Pasteur Institute }\end{array}$ \\
\hline
\end{tabular}

CDC, Centers for Disease Control and Prevention; nCoV, novel coronavirus; SARS-CoV-2, severe acute respiratory syndrome coronavirus 2; WH0, World Health Organization. 
Table 2 Oligonucleotide Sequences of Primers and Probes of Oncobit Real-Time RT-PCR and Digital Droplet PCR Protocols

\begin{tabular}{ll}
\hline Primer/probe name & Sequence \\
\hline N2 forward primer & $5^{\prime}-$ TTACAAACATTGGCCGCAAA-3' \\
N2 reverse primer & $5^{\prime}$-GCGCGACATTCCGAAGAA-3' \\
N2 probe (FAM) & $5^{\prime}$-ACAATTTGCCCCCAGCGCTTCA-3' \\
ORF1ab forward primer & $5^{\prime}$-CCCTGTGGGTTTTACACTTAA-3' \\
ORF1ab reverse primer & $5^{\prime}$-ACGATTGTGCATCAGCTGA-3' \\
ORF1ab probe (Cy5) & $5^{\prime}$-CCGTCTGCGGTATGTGGAAAGGTTATGG-3' \\
RNaseP forward primer & $5^{\prime}$-AGATTTGGACCTGCGAGCG-3' \\
RNaseP reverse primer & $5^{\prime}$-GAGCGGCTGTCTCCACAAGT-3' \\
RNaseP probe (HEX) & $5^{\prime}-$ TTCTGACCTGAAGGCTCTGCGCG-3' $^{\prime}$ \\
\hline
\end{tabular}

\section{Materials and Methods}

\section{Clinical Samples}

Patient samples were collected by nasopharyngeal and/or oropharyngeal swabs (CM-FS913, iClean, San Ramon, CA) at the University Hospital Zurich and at ADMed Laboratory in La Chaux-de-Fonds, Switzerland (Copan Diagnostics, Brescia, Italy). The non-COVID-19 samples (other respiratory disease samples) were provided by ADMed Laboratory and were selected after having been tested on the Respiratory Panel FilmArray on Biofire (bioMérieux, Marcy-l'Étoile, France). Household samples were collected by swabbing of the different surfaces in a quarantined household of a SARSCoV-2-positive patient. All swabs were stored in a viral transport medium (CDC, https://www.cdc.gov/coronavirus/ 2019-ncov/downloads/Viral-Transport-Medium.pdf, Accessed March 20, 2020) or Eswab (Copan Diagnostics, Murrieta, CA) at $4^{\circ} \mathrm{C}$ for a maximum of 48 hours or stored at $-80^{\circ} \mathrm{C}$ until further analyses. All household swabbing participants provided informed consent for the study, and both the assay establishment and household studies were approved by the Cantonal Ethics Committee (BASEC-Nr-2020-00660 and BASEC-Nr-2020-00659, respectively).

Table 3 Reaction Mix for Oncobit Real-Time RT-PCR Protocol

\begin{tabular}{lc}
\hline Reagent & $\begin{array}{c}\text { Volume per } \\
\text { reaction, } \mu \mathrm{L}\end{array}$ \\
\hline TaqPath 1-Step Multiplex Master Mix & 5 \\
$\quad$ (no ROX) (catalog number A28521, & \\
$\quad$ Thermo Fisher Scientific, Waltham, MA), 4X & \\
N2 probe (FAM) $(100 \mu \mathrm{mol} / \mathrm{L})$ & 0.05 \\
ORF1ab probe (Cy5) $(100 \mu \mathrm{mol} / \mathrm{L})$ & 0.05 \\
RNaseP probe $(\mathrm{HEX})(100 \mu \mathrm{mol} / \mathrm{L})$ & 0.05 \\
N2 forward primer $(100 \mu \mathrm{mol} / \mathrm{L})$ & 0.06 \\
N2 reverse primer $(100 \mu \mathrm{mol} / \mathrm{L})$ & 0.06 \\
ORF1ab forward primer $(100 \mu \mathrm{mol} / \mathrm{L})$ & 0.06 \\
ORF1ab reverse primer $(100 \mu \mathrm{mol} / \mathrm{L})$ & 0.06 \\
RNaseP forward primer $(100 \mu \mathrm{mol} / \mathrm{L})$ & 0.03 \\
RNaseP reverse primer $(100 \mu \mathrm{mol} / \mathrm{L})$ & 0.03 \\
Nuclease-free water & 4.55 \\
Total & 20.0 \\
\hline
\end{tabular}

\section{RNA Extraction}

Viral RNA was extracted as previously described ${ }^{5}$ using a magnetic bead-based (SpeedBeads, GE Healthcare, Darmstadt, Germany) extraction kit for the KingFisher instrument (MagMax, Thermo Fisher Scientific, Waltham, MA).

\section{Detection of SARS-CoV-2 by RT-PCR Protocols}

Four commercially available, one customized (Pasteur Institute, Paris, France), and in-house optimized RT-PCR protocols (Table 1$)^{6,7}$ were compared. Primer probes design, reaction mix, and thermal cycling conditions are given in Tables 2-4 respectively. All RT-PCR protocols were run according to manufacturer instructions on a QuantStudio 5 DX real-time PCR system (catalog number A36324, Thermo Fisher Scientific), and data were analyzed with the Design and Analysis Software DA version 2.4 (Thermo Fisher Scientific) except for the Euroimmun protocol, which was run on LightCycler 480 II (RocheDiagnostics, Basel, Switzerland). Fast cycling mode was used, and a comparative $\mathrm{Ct}$ analysis method was performed.

For the CDC protocol, an RT-PCR result was defined as inconclusive if only the $N 1$ gene ( $\pm N 3$ gene) was positive or if only the $N 2$ gene ( $\pm N 3$ gene) was positive. For the TFMultiPlex (Thermo Fisher Scientific), TF-SinglePlex (Thermo Fisher Scientific), and Oncobit protocols, an RTPCR result was considered inconclusive if only one of two or three of the viral genes was positive. Inconclusive results were not repeated. The Euroimmun protocol (Luebeck, Germany) does not have the inconclusive category.

\section{Detection of SARS-CoV-2 by ddPCR}

The ddPCR protocol for SARS-CoV-2 detection targets two viral genomic regions of the SARS-CoV-2 gene (ORFlab and N2) and uses the human RNase P gene as an in-process control. The following probes for the three genes were used: ORFIab (FAM and HEX), N2 (FAM), and RNase $P$ (HEX) (Table 2). Briefly, $20 \mu \mathrm{L}$ of reaction mix (containing 1-Step RT-ddPCR Advanced Kit for Probes Mastermix; BioRad, Luxembourg, Luxembourg) was combined with $10 \mu \mathrm{L}$ 
Tastanova et al

\begin{tabular}{llll}
\hline Table $\mathbf{4}$ & Thermal Cycling Conditions for Oncobit Real-Time RT-PCR Protocol & & \\
\hline Stage & Step & Temperature, ${ }^{\circ} \mathrm{C}$ & Time \\
\hline Hold & UNG incubation & 25 & 2 minutes \\
Hold & Reverse transcription & 53 & 10 minutes \\
Hold & Activation & 95 & 2 minutes \\
Cycling (40 cycles) & Denaturation & 95 & 3 seconds \\
& Anneal/extension & 60 & 30 seconds \\
\hline
\end{tabular}

of RNA sample for a final reaction volume of $30 \mu \mathrm{L}$. The final concentrations were $90 \mathrm{nmol} / \mathrm{L}$ for primers $($ ORFlab,$N 2$, $R N a s e P), 19.5 \mathrm{nmol} / \mathrm{L}$ for $R d R P$ probes, $30 \mathrm{nmol} / \mathrm{L}$ for the $N 2$ probe, and $40 \mathrm{nmol} / \mathrm{L}$ for the RNase $P$ probe. The SARSCoV-2 Positive Run Control (catalog number COV019CE, Bio-Rad) was used as positive control. ddPCR was run ac[T5] cording to the program listed in Table 5 using QX200 Droplet Digital PCR System (Bio-Rad). The swabbing household samples from a laptop, newspaper, or door handle as well as the nontemplate control were tested in two independent runs.

\section{LoD, Sensitivity, and Specificity Calculation}

The LoD of four published SARS-CoV-2 detection protocols (CDC, TF-MultiPlex, TF-SinglePlex, and Euroimmun) was determined using a dilution of an external quality assessment quantitative test sample (Instand, https://www.instand-ev.de/en/news/detail/news/ne uartiges-coronavirus-sars-cov-2-2019-ncov-im-vorgezoge nen-instand-ringversuch-virusgenom-nachw/?tx_news_pi $1 \% 5$ Bcontroller $\% 5 D=$ News\&tx_news_pil $\% 5$ Baction\% $\%$ $D=$ detail $\&$ cHash $=$ f91865b86af167390788c7f404b16e $7 e$, last accessed November 12, 2020). Linear regression was used to determine the line of best fit for the relationship between $\mathrm{Ct}$ and viral copies. A Ct value of 40 was set as the minimum amount of viral copies detected by RT-PCR. LoD for Oncobit ddPCR protocol was determined using a dilution of the SARS-CoV-2 Positive Run Control (catalog number COV019CE, Bio-Rad).

For sensitivity and specificity value calculations of each assay, the results of RT-PCR obtained from the ADMed Laboratory were used as the gold standard reference. The sensitivity was defined with the formula $\mathrm{TP} /(\mathrm{TP}+\mathrm{FN})$, whereas specificity was defined as TN/(TP $+\mathrm{FP})$, where TP indicates true positive, FP indicates false positive, TN indicates true negative, and FN indicates false negative. If the result of tested assays matched the reference, it was labeled

Table 5 Thermal Cycling Conditions for Oncobit Digital Droplet PCR Protocol

\begin{tabular}{lll}
\hline Stage & Temperature, ${ }^{\circ} \mathrm{C}$ & Time \\
\hline Hold & 50 & 60 minutes \\
Hold & 95 & 10 minutes \\
Cycling (55 cycles) & 95 & 30 seconds \\
& 59 & 1 minute \\
Hold & 98 & 10 minutes \\
Hold & 4 & 1 minute \\
\hline
\end{tabular}

concordant. If the result from the tested assays did not match the gold reference, it was labeled discordant. Inconclusive results were excluded from sensitivity and specificity calculations.

\section{SARS-CoV-2 Infectivity Assay}

The viral infectivity assay was performed as previously described $^{8-10}$ with slight modifications. Briefly, $5 \times 10^{4}$ Vero E6 cells (catalog number CCL-81, ATCC, Manassas, VA) were seeded on 96-well flat bottom cell culture plates in $200 \mu \mathrm{L}$ of high glucose Dulbecco's modified Eagle's medium medium supplemented with L-glutamate, sodium pyruvate, $\mathbf{Q}^{7}$ nonessential amino acids, HEPES, 5\% fetal cow serum, and Normocin (catalog number ant-nr-1, InvivoGen, Toulouse, France). After 24 hours of incubation $\left(37^{\circ} \mathrm{C}, 5 \% \mathrm{CO}_{2}\right)$, the medium was removed, and $100 \mu \mathrm{L}$ of a virus test solution or the positive SARS-CoV-2 control (provided by Prof. Volker Thiel, Inst. Virology \& Immunology, University of Berne, Switzerland) was added in twofold serial dilutions to the cells. The plates were incubated for 48 hours at $37^{\circ} \mathrm{C}$. The cells were then fixed with $10 \%$ formaldehyde solution for $15 \mathrm{mi}$ nutes at room temperature, rinsed with phosphate-buffered saline, and stained with $1 \%$ crystal violet stain solution (catalog number 252532.1211, Pan Reac AppliChem, Darmstadt, Germany) for 15 minutes at room temperature. The staining solution was removed, the cells were rinsed twice with phosphate-buffered saline, and the plates dried at room temperature before assessment for viral plaques.

\section{Results}

\section{Description and Comparison of SARS-CoV-2 RT-PCR Detection Protocols}

The six RT-PCR protocols compared in this study use the same principle of isolating viral RNA from the nasopharyngeal and/or oropharyngeal swabs or bronchial fluid and running a 1-step RT reaction followed by real-time amplification of two or three SARS-CoV-2 target genes (Figure 1). [F1] Summary and comparison of all tested RT-PCR protocols is given in Table 6. All protocols have internal controls, nontemplate controls and positive controls. In TF-MultiPlex, the phage MS2 is added as the internal control that serves as both RNA isolation and reaction control. All other protocols except for Euroimmun (where the type of interncal control is not indicated) use a widely accepted reaction control RNAseP to ensure that RNA isolation worked and RT-PCR reaction 
Benchmarking COVID-19 Detection Methods

was not inhibited. The protocol design is single plex, double plex, or multiplex. Euroimmun protocol stands out with its design, with two target probes coupled to the same reporter color FAM. The viral RNA input is 5 to $10 \mu \mathrm{L}$. Because of unspecific E-gene amplification (Supplemental Table S1), the protocol developed by Pasteur Institute was not used further in this comparative study.

\section{LoD of Real-Time RT-PCR and ddPCR SARS-CoV-2 Detection Protocols}

With a Ct value cut-off of 40, the five RT-PCR SARS-CoV2 detection protocols (CDC, TF-MultiPlex, TF-SinglePlex, Euroimmun, and Oncobit) as well as the Oncobit ddPCR protocol had an LoD between 1 and 2 viral copies $/ \mu \mathrm{L}$ [F2] (Figure 2, A and B). Values $<1$ copy $/ \mu \mathrm{L}$ indicate high sensitivity of the tested protocol (Figure 2, A and B).

\section{Specificity and Sensitivity of Real-Time RT-PCR SARS- CoV-2 Detection Protocols}

For the sensitivity and specificity of the SARS-CoV-2 detection protocols (CDC, TF-SinglePlex, TF-MultiPlex, Euroimmun, and Oncobit), a cohort of 92 SARS-CoV2-positive samples and 92 SARS-CoV-2-negative samples was used that were provided by ADMed Laboratory. A comparison to SARS-CoV-2-positive results showed similar sensitivity of all tested protocols, with a 93.6\% sensivity for TF-SinglePlex and $96.7 \%$ to $97.8 \%$ sensitivity for the other protocols (Figure 3A). In the specificity cohort, 22 samples had a confirmed diagnosis of other respiratory diseases (Supplemental Table S2), and 70 samples tested negative for all listed respiratory diseases, including SARS-CoV-2. All protocols, except TF-SinglePlex, had no cross-reactivity (Figure 3A), including samples that tested positive for four other

Table 6 Comparative Overview of Six Real Time RT-PCR Protocols

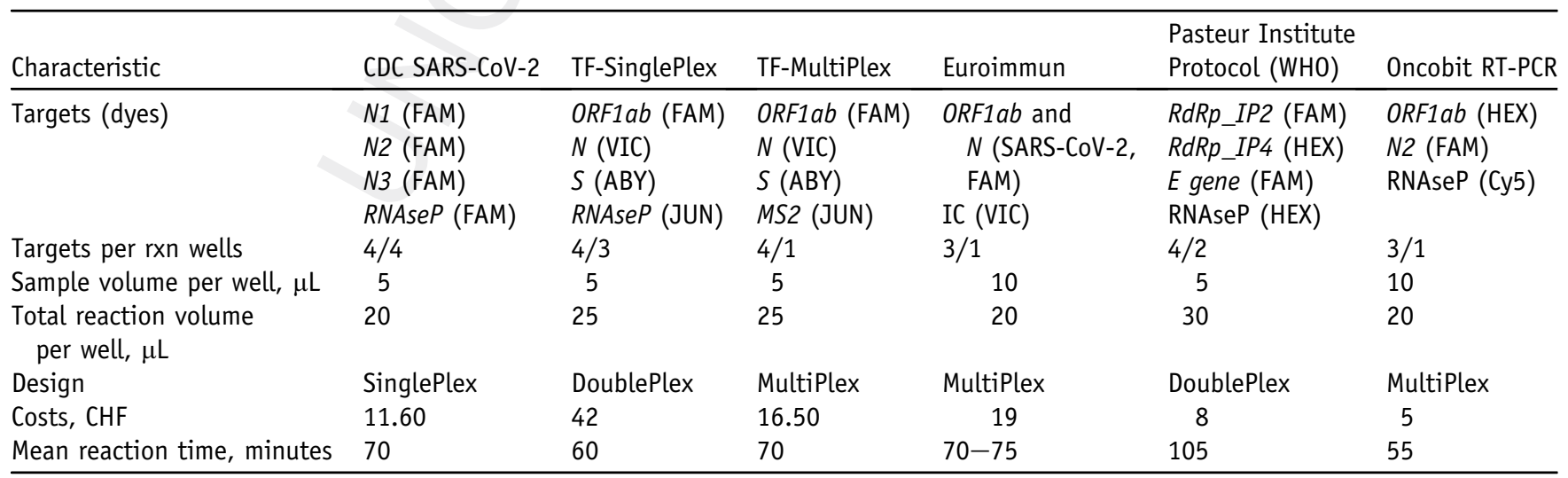

CDC, Centers for disease control and prevention; IC, internal control; rxn, ; SARS-CoV-2, severe acute respiratory syndrome coronavirus 2; WH0, World Health Organization. 

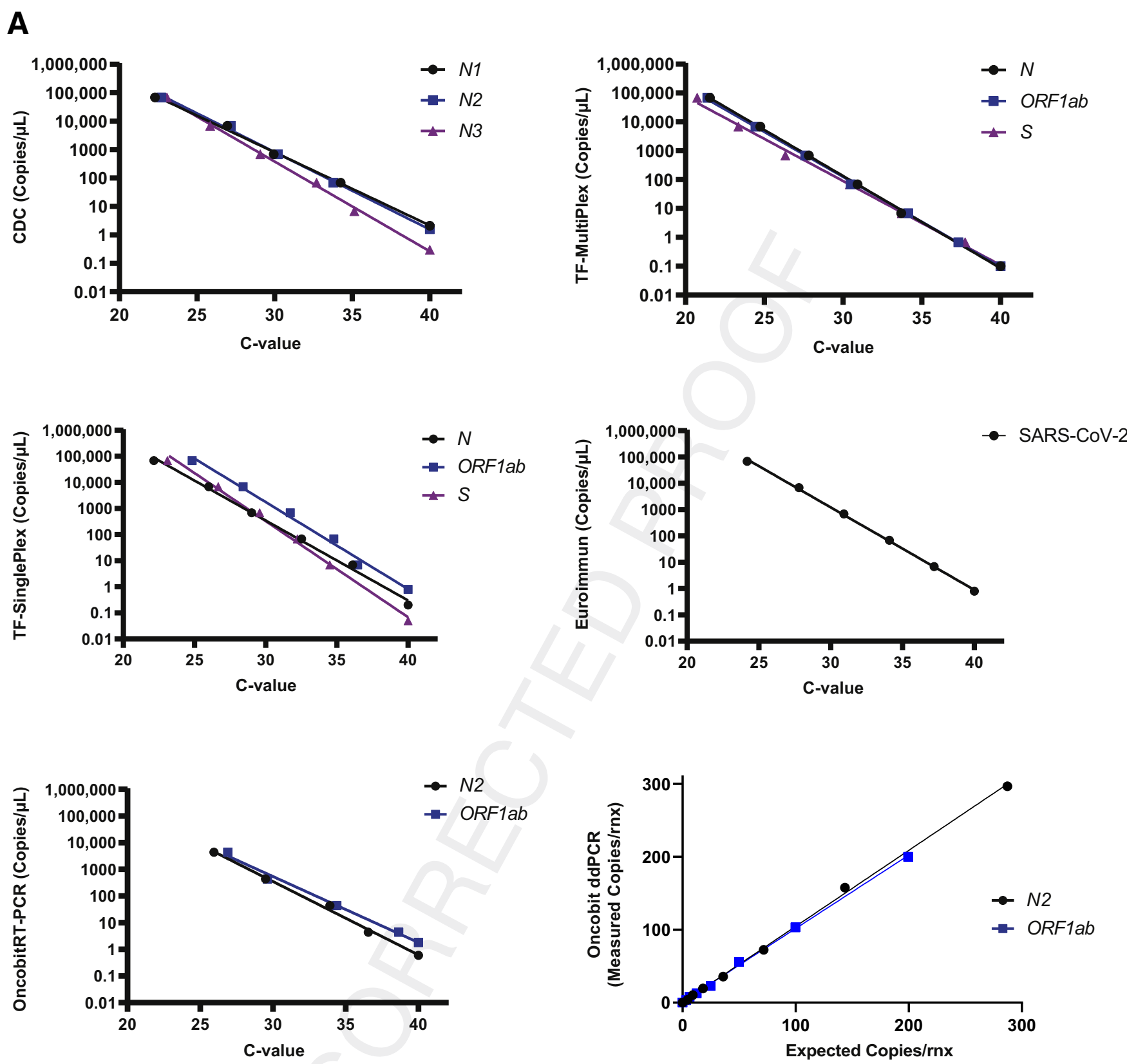

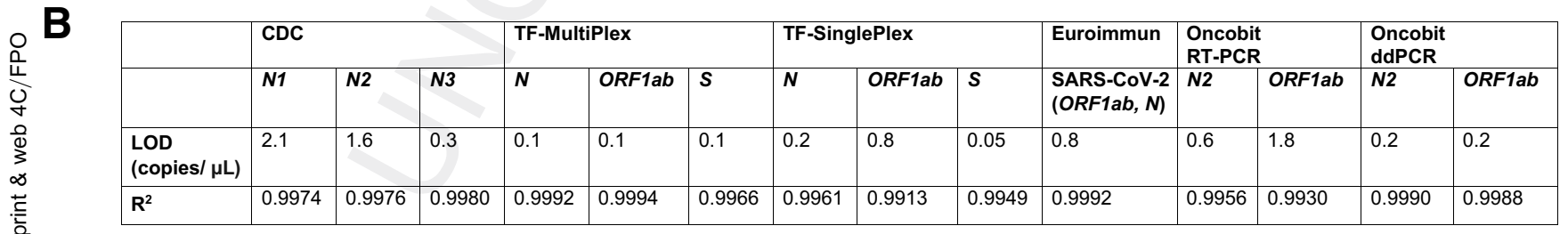

Figure 2 Limit of detection (LoD) of real-time RT-PCR and digital droplet PCR (ddPCR) severe acute respiratory syndrome coronavirus 2 (SARS-CoV-2) detection protocols. A: LoD (viral copies per microliter) of different target genes of Centers for Disease Control and Prevention (CDC), TF-SinglePlex, TF-MultiPlex, Euroimmun, Oncobit RT-PCR, and Oncobit ddPCR SARS-CoV-2 detection protocols. B: Calculated $R^{2}$ values of SARS-CoV-2 detection protocols. rxn,

Inconclusive results were found in $0.5 \%$ to $3.2 \%$ of these types of coronaviruses (Supplemental Table S2). The specificity was thus $100 \%$ for all protocols except for TF-SinglePlex, which had a specificity of $98.7 \%$ (Figure 3A).
184 samples, with TF-MultiPlex and Oncobit providing the most accuracy (Figure 3A). Comparing RT-PCR results (positive, negative, or inconclusive) of all 184 samples, the
683

684

685

686

687

688

689

690

691

692

693

694

695

696

697

698

699

700

701

702

703

704

705

706

707

708

709

710

711

712

713

714

715

716

717

718

719

720

721

722

723

724

725

726

727

728

729

730

731

732

733

734

735

736

737

738

739

740

741

742

743

744 
Benchmarking COVID-19 Detection Methods

B

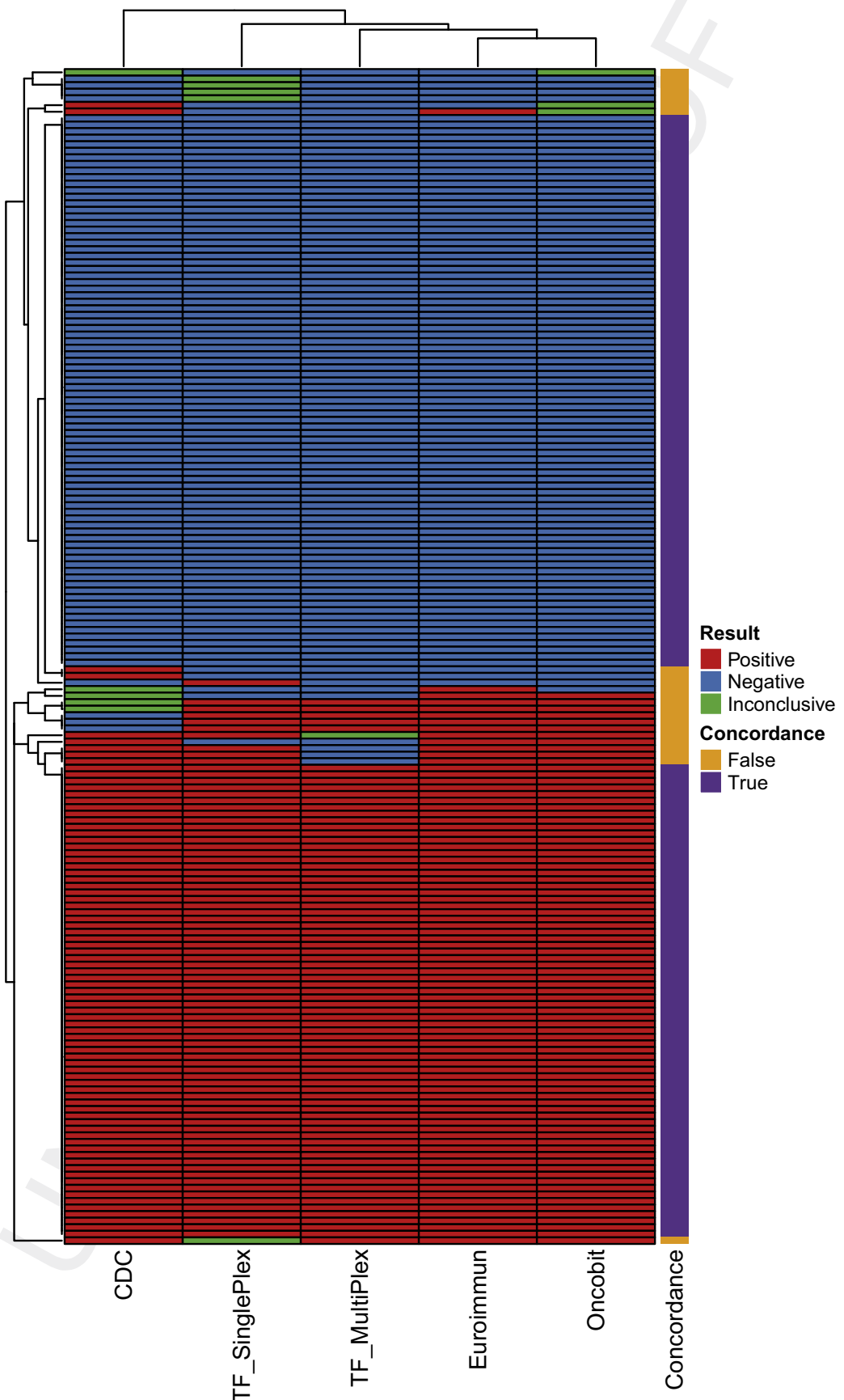

Figure 3 Specificity and sensitivity of real-time RT-PCR severe acute respiratory syndrome coronavirus 2 (SARS-CoV-2) detection protocols. A: Performance calculation (sensitivity/specificity) as well as calculation of percentage of inconclusive results of five real-time RT-PCR detection protocols [Centers for Disease Control and Prevention (CDC), TF-SinglePlex, TF-MultiPlex, Euroimmun, and Oncobit]. The Euroimmun RT-PCR detection protocol does not have the inconclusive category; inconclusive for Euroimmun equals an invalid result. B: Heatmap summarizing concordance of five real-time RT-PCR detection protocols (CDC, TFSinglePlex, TF-MultiPlex, Euroimmun, and Oncobit) for both sensitivity (bottom) and specificity (top) sample cohorts. 


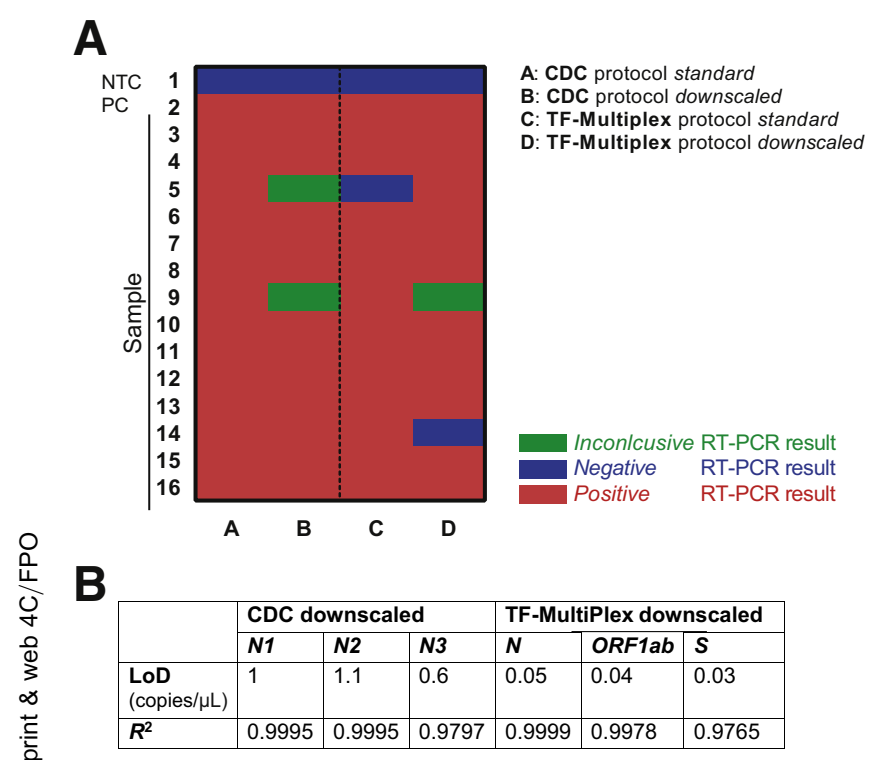

Figure 4 Downscaling of the Centers for Disease Control and Prevention (CDC) and TF-MultiPlex protocols. A: Heatmap summarizing results of standard and downscaled protocol (CDC and TF-MultiPlex). For the CDC protocol, a RT-PCR result was defined inconclusive if RT-PCR was positive for only $N 1( \pm N 3)$ or for only N2 $( \pm N 3)$. For TF-MultiPlex a RT-PCR result was considered inconclusive if only one of the viral genes was positive. B: Limit of detection (LoD) (copies per microliter) and $R^{2}$ values of downscaled protocols (CDC and TF-MultiPlex). NTC, nontemplate control; $\mathrm{PC}$, positive control.

overall nonconcordance between all the protocols was $14.7 \%$ (Figure 3B).

\section{Optimization of Testing Capacity}

To optimize testing capacity, recommended reaction volumes in commercial protocols were downscaled, and published primer/probe sequences were customized to have an in-house developed protocol (Oncobit). Using a previously confirmed SARS-CoV-2-positive cohort of 14 samples, we compared the CDC and TF-MultiPlex protocols with recommended reaction volume and reaction volumes reduced by $50 \%$. RNA sample input was always the same. The Oncobit protocol was the cheapest (Table 6), had the shortest RT-PCR reaction time requirement (Table 6), and had the most reliable access to consumables (Microsynth, Balgach, Switzerland). The specificity and sensitivity of the Oncobit protocol were comparable with other commercial SARS-CoV-2 RT-PCR detection kits (Figure 3, A and B).

A downscaled CDC protocol showed two (14.3\%) inconclusive results, a standard TF-MultiPlex protocol showed one $(7.1 \%)$ false-negative result, and a downscaled TF-MultiPlex protocol revealed one (7.1\%) false-negative [F4] as well as one (7.1\%) inconclusive result (Figure 4A). Furthermore, LoD of downscaled CDC and TF-MultiPlex protocols showed a sensitivity of 1 copy $/ \mu \mathrm{L}$ with a $\mathrm{Ct}$ value cut-off of 40 (Figure 4B).
Application of SARS-CoV-2 Detection Protocols on Swabbed Surfaces

Having compared and established the RT-PCR protocols for SARS-CoV-2 diagnostics, the possibility of application of the RT-PCR and ddPCR protocol for SARS-CoV-2 detection on environmental samples was examined. Swabs of different surfaces from a SARS-CoV-2 quarantined household were collected and analyzed by two validated RT-PCR protocols. In addition, an in-house ddPCR protocol was developed to accurately detect and quantify virus.

On the day of household surface swabbing (April 25, 2020) of the SARS-CoV-2-positive family, only patient 2 was swabbed again and tested positive but reported no symptoms (Supplemental Figure S1A). The pharyngeal swab as well as the swabbed surface samples were collected on the same day and tested with three different SARS-CoV2 detection protocols (CDC, TF-MultiPlex, and ddPCR). The pharyngeal swab tested positive (cycle thresholds $>30$ ) on three different protocols. The laptop keyboard and two more swabbed surface (the door handle and newspaper) samples had positive and inconclusive results, respectively (Supplemental Table S3), whereas no infectivity for any of the samples was detected (Supplemental Figure S1B).

\section{Discussion}

Real-time RT-PCR remains the most sensitive method for early detection of SARS-CoV-2. We report a comparison of LoD, specificity, sensitivity, economic, and practical advantages of four commercial SARS-CoV-2 detection kits as well as one optimized in-house RT-PCR SARS-CoV-2 protocol. A study comparing RT-PCR with rapid fluorescence immunochromatographic assay-based SARS-CoV-2 nucleocapsid protein antigen detection method showed that sensitivity of the rapid method was only approximately $75.6 \%{ }^{11}$; therefore, RT-PCR remains a more sensitive detection method for SARS-CoV-2. Most of the reported multiplatform comparison studies on real-time RT-PCR SARS-CoV-2 detection performed the benchmarking only on a limited number of samples and tested only commercial detection kits, ${ }^{10,12,13}$ and some studies limited the comparison only to sensitivity assessment. ${ }^{14}$

In this study, a low LoD and high sensitivity for four commercial SARS-CoV-2 RT-PCR detection protocols were observed by using standard quantitative test samples and a cohort of 92 SARS-CoV-2-positive samples, respectively. Furthermore, specificity of those protocols was tested and confirmed with 92 samples that had confirmed SARS-CoV-2-negative result or were collected in prepandemic times from patients presenting with respiratory symptoms (Supplemental Table S2).

In addition, downscaling of two commercial protocols that were chosen for the diagnostic routine (CDC and TF- 
MultiPlex) could be an option to save resources. This downscaling is especially important in times when a high demand for SARS-CoV-2 testing causes supply chain problems as occurred at the beginning of the pandemic in Europe. As an alternative strategy to optimize costs and increase testing capacity, an in-house protocol was developed in collaboration with the diagnostics company Oncobit by adapting previously published primer sequences for multiplex analysis. The customized Oncobit protocol was the least costly and fastest protocol when compared with other commercial RT-PCR protocols tested in this study.

To expand the application of RT-PCR-based detection protocols, a testing of swabbed surfaces from a SAR-CoV2 quarantined household was performed. Results showed that RT-PCR protocols detected the viral genetic material on the laptop keyboard, and this result was confirmed by a more sensitive ddPCR method. Two more surfaces showed inconclusive results (a newspaper and a door handle, with viral copies detectable by ddPCR, however below the LoD) (Supplemental Table S3). Nasopharyngeal swab taken on the same day tested positive; however, infectivity assay for all samples showed negative results. These findings demonstrate the possibility of applying the RTPCR-based protocols on nonpatient samples that could be of use for larger environmental studies. Summarizing the comparative study, we found that most commercial and customized RT-PCR-based detection protocols are highly effective at detecting viral presence in classic nasopharyngeal and/or oropharyngeal swabs, and because of its high sensitivity, RT-PCR-based detection protocols can be applied to the testing of environmental samples.

\section{Acknowledgments}

We thank Gaetana Restivo for help with obtaining ethical clearance for the study. We are very grateful to Jan Kaesler, Mirka Schmid, Muriel Traexler, Melanie Maudrich, and the entire Dermatology biobank team of University Hospital Zurich for big help with running the experiments and technical help.

\section{Author Contributions}

The idea of the study was conceived by M.P.L., P.P.B., A.T., C.I.S., A.Dz., and P.F.C. Experimental design was performed by M.P.L., P.P.B., A.T., C.I.S., E.B., A.Dz., and P.J. The experiment was executed by A.T., C.I.S., E.B., A.Dz., P.J., and A.D. Data analysis was performed by C.I.S., P.F.C., A.T., A.Dz., E.B., and P.J. The study was supervised by M.P.L. and P.P.B. The manuscript was drafted by A.T. and C.I.S. All authors edited and contributed into manuscript writing.

\section{Supplemental Data}

Supplemental material for this article can be found at http://doi.org/10.1016/j.jmoldx.2021.04.009.

\section{References}

1. Petersen E, Koopmans M, Go U, Hamer DH, Petrosillo N, Castelli F, Storgaard M, Al Khalili S, Simonsen L: Comparing SARS-CoV-2 with SARS-CoV and influenza pandemics. Lancet Infect Dis 2020, 20: e238-e244

2. Balabdaoui F, Mohr D: Age-stratified discrete compartment model of the COVID-19 epidemic with application to Switzerland. Sci Rep 2020, 10:21306

3. Bi J, Lin Y, Zhong R, Jiang G, Verma V, Shi H, Li J, Tong X, Li Y, Hu D, Liang W, Han G, He J: Prevalence and clinical characterization of cancer patients with asymptomatic SARS-CoV-2 infection history. J Infect 2020, 81:e22-e24

4. Oran DP, Topol EJ: Prevalence of asymptomatic SARS-CoV-2 infection: a narrative review. Ann Intern Med 2020, 173:362-367

5. Eichhoff OM, Bellini E, Lienhard R, Stark WJ, Bechtold P, Grass RN, Bosshard PP, Levesque MP: Comparison of RNA extraction methods for the detection of SARS-CoV-2 by RT-PCR. medRxiv 2020. doi: 10.1101/2020.08.13.20172494

6. Corman VM, Landt O, Kaiser M, Molenkamp R, Meijer A, Chu DK, Bleicker T, Brunink S, Schneider J, Schmidt ML, Mulders DG, Haagmans BL, van der Veer B, van den Brink S, Wijsman L, Goderski G, Romette JL, Ellis J, Zambon M, Peiris M, Goossens H, Reusken C, Koopmans MP, Drosten C: Detection of 2019 novel coronavirus (2019-nCoV) by real-time RT-PCR. Euro Surveill 2020, $25: 2000045$

7. Dong L, Zhou J, Niu C, Wang Q, Pan Y, Sheng S, Wang X, Zhang Y, Yang J, Liu M, Zhao Y, Zhang X, Zhu T, Peng T, Xie J, Gao Y, Wang D, Dai X, Fang X: Highly accurate and sensitive diagnostic detection of SARS-CoV-2 by digital PCR. Talanta 2021, 224:121726

8. Aoki-Utsubo C, Chen M, Hotta H: Virucidal and neutralizing activity tests for antiviral substances and antibodies. Bio-protocol 2018, 8: e2855

9. Wu F, Wang A, Liu M, Wang Q, Chen J, Xia S, Ling Y, Zhang Y, Xun J, Lu L, Jiang S, Lu H, Wen Y, Huang J: Neutralizing antibody responses to SARS-CoV-2 in a COVID-19 recovered patient cohort and their implications. medRxiv 2020. doi:10.1101/2020.03.30.20047365

10. Chu H, Chan JF, Yuen TT, Shuai H, Yuan S, Wang Y, Hu B, Yip CC, Tsang JO, Huang X, Chai Y, Yang D, Hou Y, Chik KK, Zhang X, Fung AY, Tsoi HW, Cai JP, Chan WM, Ip JD, Chu AW, Zhou J, Lung DC, Kok KH, To KK, Tsang OT, Chan KH, Yuen KY: Comparative tropism, replication kinetics, and cell damage profiling of SARS-CoV-2 and SARS-CoV with implications for clinical manifestations, transmissibility, and laboratory studies of COVID-19: an observational study. Lancet Microbe 2020, 1:e14-e23

11. Diao B, Wen K, Zhang J, Chen J, Han C, Chen Y, Wang S, Deng G, Zhou H, Wu Y: Accuracy of a nucleocapsid protein antigen rapid test in the diagnosis of SARS-CoV-2 infection. Clin Microbiol Infect 2021, 27:289.e1-289.e4

12. Igloi Z, Leven $\mathrm{M}$, Abdel-Karem Abou-Nouar Z, Weller B, Matheeussen V, Coppens J, Koopmans M, Molenkamp R: Comparison of commercial realtime reverse transcription PCR assays for the detection of SARS-CoV-2. J Clin Virol 2020, 129:104510

13. van Kasteren PB, van der Veer B, van den Brink S, Wijsman L, de Jonge J, van den Brandt A, Molenkamp R, Reusken C, Meijer A: Comparison of seven commercial RT-PCR diagnostic kits for COVID19. J Clin Virol 2020, 128:104412

14. Zhen W, Manji R, Smith E, Berry GJ: Comparison of four molecular in vitro diagnostic assays for the detection of SARS-CoV-2 in nasopharyngeal specimens. J Clin Microbiol 2020, 58:e0743-20 
Supplemental Figure S1 Patients Ct values and symptom progression as well as infectivity examination of swabbed patient and surface samples. A: The patient's Ct values and symptom progression. Mean of Ct values of the N1, N2, and N3 viral genes are shown (Centers for Disease Control and Prevention protocol). Patient 1 was a 42-year-old woman; patient 2, 42-year-old man; patient 3, 6-year-old boy; and patients 4, 4-year-old boy. The Ct values at the time of diagnosis for patient 1 are missing because the patient was tested in a different laboratory. On April 7, 2020, the patient experienced shortness of breath, which lasted for 3 days. On April 7, 2020, the 42-year-old man (father, patient 2) tested positive for severe acute respiratory syndrome coronavirus 2 (SARSCoV-2), with symptoms resolving after 10 days. As the symptoms gradually resolved, we observed increasing $\mathrm{Ct}$ values in both patients. On April 12 , the two children (patients 3 and 4) also tested positive for SARS-CoV-2 but remained asymptomatic at all times. Family members in the household were swabbed on the April 25, with patients being asymptomatic for at least 2 days. B: An infectivity assay using Vero E6 cells found no plaque formation for any of the samples that tested positive by real-time RT-PCR (patient's throat, laptop keyboard, newspaper, and toilet rim).

1125

1126 